\title{
Damping Matrix Identification for Structural Health Monitoring Considering Sensitivity of Initial Unknown Covariance
}

\author{
Mohammad S. Miah', Michael Kaliske ${ }^{1}$, Md Jihad Miah ${ }^{2}$ \\ ${ }^{1}$ Faculty of Civil Engineering, Dresden University of Technology- TU Dresden, 01187 Dresden, Germany \\ mohammad_shamim.miah@tu-dresden.de; michael.kaliske@tu-dresden.de \\ ${ }^{2}$ Department of Civil Engineering, University of Asia Pacific, Dhaka-1215, Bangladesh \\ jihad.miah@uap-bd.edu
}

\begin{abstract}
The use of smart material and systems are gaining serious attention in the area of structural engineering due to their superior performance in real-world applications. The aforementioned technology requires real-time feedback in order to monitor them safely. This problem even gets into more complicated situation when it comes to adjust the current uncertainty of structures. To do this end, the realtime parameter estimation as well as state update (e.g. displacement and velocity) of an eight-storey frame type structure is considered. To deal with the early mentioned nonlinear problem due to multiple unknown variables a nonlinear observer is employed, namely, the unscented Kalman filter (UKF). As the parameter estimation along with the sate update are not that straight forward task due to the sensitivity of the initial covariance of the unknown parameters as well as for states uncertainties. The outcome shows that the proposed approach is capable of estimating the unknown parameters (e.g. damping matrix) quite efficiently with high accuracy. The investigated approach will facilitate the overall health monitoring of dynamical systems.
\end{abstract}

Keywords: Structural health monitoring; Dynamic loads; Structural damping; System identification.

\section{Introduction}

The estimation of structural responses such as displacements and velocities are essential to understand the behaviour of any dynamical system. The discussed issue can be resolved by solving second order differential equations so-called the equation of motions. However, when the structural parameters such as stiffness or damping are not entirely known forms a nonlinear problem. Therefore, in order to estimate states and unknown structural parameters, the early mentioned nonlinear problem needs to be solved [1]. System identification is a new trend in the area of civil engineering as many of the recent structures are coupled with complex systems and monitoring schemes. As numerous uncertainties are associated to the aforementioned task which makes the parameter identification work non-trivial [2]-[3]. The associated complexity can be induced by the sensitivity of states, system noises, process and measurement noise as well as unknown parameters. Additionally, the uncertainties are coming from different sources, for instance, form the measurement sensors as well as modelling errors.

The usefulness of the real-time parameters/system identification has been reported by many researchers such as [4]-[5][7] for various applications. For instance, in [2] investigated the possibility of system identification by using a minicomputer. While in [5], the author monitored the deformation via the use of Global Positioning System (GPS) and Kalman filter. In [6], has reported the possibility of real-time system identification of nonlinear system and [7] performed an experimental verification of a newly developed system identification and monitoring schemes in real-time. It is essential to monitor the structures in real-time to avoid any unexpected changes those might be crucial for its safety. Not only dynamic loads such as earthquake, wind load, temperature variation but also service load may cause serious damage. Therefore, the identification of structural unknown properties somewhat substantial for tracking/assessing its health.

The identification of stiffness components would be simpler task in comparison to the identification of damping of a system. It is always a complicated task to deal with damping as it shows quite complex behaviour. This study investigates the parameter identification of an eight-storey frame by employing extremely devastation type harmonic load. To do this herein, the structure was combined with a nonlinear observer namely the unscented Kalman filter (UKF). And the sensitivity of initial covariance is studied by considering state estimation and parameters identification simultaneously. 
The rest of the paper is organized as: Section 2 describes the studied problem including basic mathematical formulations. Section 3 presents brief overview of the nonlinear observer. Section 4 contains the numerical investigations. Finally, the last part of the paper summarized the outcome of the study.

\section{Description of the Problem}

In order to perform numerical investigations, an eight storey frame type structure is considered. For avoiding complexity, the dynamical model is assumed to be modelled as mass-spring-dashpot system. Hence, the system properties such as mass, damping, and stiffness matrices size would be 8-by-8 and the displacement, velocity, and acceleration vector size would be 8 -by-1. And in order to perform numerical investigations in a nearly real-time platform, the SIMULINK platform was combined with MATLAB ${ }^{\circledR}$. It needs to be mentioned that all of the variables indicated in the early mentioned figures are explained later in this section. The dynamical structure is assumed to be described by mass-spring-dashpot system. And the aforementioned problem can be described by the equation of motion given below

$$
M \ddot{Z}(t)+C \dot{Z}(t)+K Z(t)=M^{-1} \gamma f_{w}
$$

where $t$ represents the time vector, $M, C$, and $K$ are the mass damping and stiffness matrices respectively, all of the aforementioned matrices have the size of $8 \times 8$, the displacement vector $\left.Z=\begin{array}{llllllll}z_{1} & z_{2} & z_{3} & z_{4} & z_{5} & Z_{6} & Z_{7} & z_{8}\end{array}\right\}^{T}$, while the velocity vector is given by $\dot{Z}=\left\{\begin{array}{lllllllllllllllll}\dot{z}_{1} & \dot{z}_{2} & \dot{z}_{3} & \dot{z}_{4} & \dot{z}_{5} & \dot{z}_{6} & \dot{z}_{7} & \dot{z}_{8}\end{array}\right\}^{T}$ and $\ddot{Z}=\left\{\begin{array}{llllllll}\ddot{z}_{1} & \ddot{z}_{2} & \ddot{z}_{3} & \ddot{z}_{4} & \ddot{z}_{5} & \ddot{z}_{6} & \ddot{z}_{7} & \ddot{z}_{8}\end{array}\right\}^{T}$ represents the acceleration, $f_{w}$ represents the input excitation, and $\gamma$ is the input excitation location control vector.

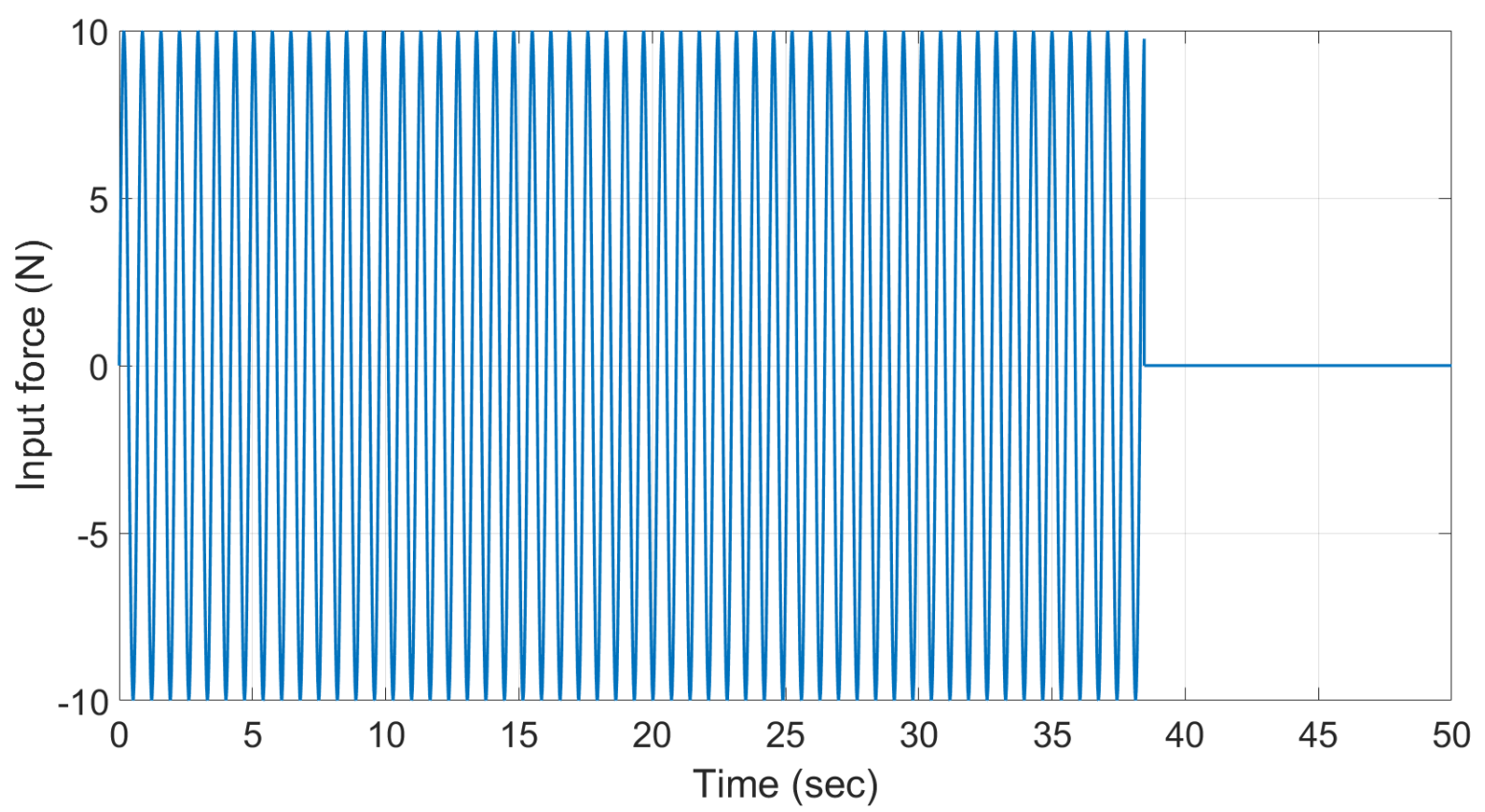

Fig. 1: The input excitation.

The simulations are performed by employing the harmonic type input excitation. Additionally, the input force was amplified 10 times, means $\delta=10$. The discussed input excitation is described via the equation given by,

$$
f_{w}(t)=\delta \sin (2 \pi \omega t)
$$


where $f_{w}$ indicates the input excitation, $\delta$ is the amplitude of the input excitation, $\omega$ represents the excitation frequency, $t$ means the time vector. The input excitation is given in Figure 1 for the visualization purpose.

In order to avoid complexity, a compact formulation namely the state-space formulation is adopted. The first equation is called the system equation that contains mass, stiffness, damping, control force (if there is any damper into the system) information as well as exogenous input force information. The process equation is given by,

$$
\dot{X}(t)=\left[\begin{array}{cc}
0_{8 \times 8} & I_{8 \times 8} \\
-M^{-1} K & -M^{-1} C
\end{array}\right] X(t)+\left[\begin{array}{c}
0_{8 \times 1} \\
-\gamma M^{-1}
\end{array}\right] u(t)
$$

where $X$ is the state vector, $u$ represents the input force, and rest of the variable remain same as before.

And the measurement equation needs to be adjusted according to the desire. For instance, herein the accelerations are measured hence the equation was modified as:

$$
Y(t)=\alpha\left[\begin{array}{ll}
-M^{-1} K & -M^{-1} C
\end{array}\right] X(t)+D u(t)
$$

where $D$ indicates the feedthrough matrix, $\alpha$ is the location indicator vector of accelarometers and the rest of the variables remain same as before.

It needs to be mentioned that the Eqs. (3-4) are nonlinear hence it is not possible to solve them via the linear observer such as linear Kalman filer (KF). Therefore, in order to solve the discussed problem a nonlinear observer namely the unscented Kalman filter (UKF) is employed. And the formulation of the UKF is briefly discussed in the next section. To perform the system identification of the early mentioned system the process Eq. (3) needs to modify accordingly. It needs to be noted that the modified equation has one extra column where the unknown parameters are assumed to be incorporated. In this case, the full damping matrix is assumed to be unknown.

$$
\overline{\dot{X}}(t)=\left[\begin{array}{ccc}
0_{8 \times 8} & I_{8 \times 8} & 0_{8 \times 8} \\
{\left[-M^{-1} K\right]} & {\left[-M^{-1} C\right]} & 0_{8 \times 8} \\
0_{8 \times 8} & 0_{8 \times 8} & 0_{8 \times 8}
\end{array}\right] \bar{X}(t)+\left[\begin{array}{c}
0_{8 \times 1} \\
-\gamma M^{-1} \\
0_{8 \times 1}
\end{array}\right] u(t)
$$

The above problem is solved by employing a nonlinear observer known as the unscented Kalman filter (UKF). Therefore, a brief information about UKF is presented in the next section for better understanding.

\section{The Nonlinear Observer}

It can be seen from the above modified process equation the problem forms a nonlinear problem that requires a nonlinear observer. Hence, herein the unscented Kalman filter (UKF) is adopted as a nonlinear observer due to its superior performances. In [1] shows the superior performance of UKF for dealing uncertainties (considering similar properties) than linear KF. Further, an experimental validation of the previously developed methodology (e.g. [1]) was reported in [7]. The fundamentals of the UKF relies on a Gaussian random distribution those can be modelled by Sigma Points (SP). The SP follows a statistical process known as the Unscented Transformation which was proposed by Jeffrey Uhlmann in 1995. Further detail regarding Unscented Transformation can be obtained via the original work which can be obtained via [8]. Later the aforementioned work was extended which was reported in [9]. The process $(f)$ and observation $(h)$ equations are in this case reformulated for the general case of a nonlinear system as,

$$
\left\{\begin{array}{c}
\bar{X}_{k+1}=f\left(\bar{x}_{k}, u_{k}, w_{k}\right) \\
\bar{Y}_{k}=h\left(\bar{x}_{k}, u_{k}, v_{k}\right)
\end{array}\right.
$$


where $\bar{X}_{k+1}$ is the step-ahead predicted states, $\bar{x}_{k}$ represents the current states, $w_{k}$ is the process noise component, $v_{k}$ indicates the measurement noise, $\bar{Y}_{k}$ is the measure/observed response. By following the Unscented Transform at time $t_{\mathrm{k}}$, the augmented state vector is given by $\overline{\mathbf{x}}_{n-1}^{j}=\left[\overline{\mathbf{x}}_{n-1}^{T}, \mathbf{w}_{n-1}^{T}, \mathbf{v}_{n-1}^{T}\right]^{T}$.

And the Sigma Point $(\xi)$ is described as:

$$
\xi_{n-1}=\left[\begin{array}{lll}
\hat{\mathbf{x}}_{n-1}^{j} & \hat{\mathbf{x}}_{n-1}^{j}+\sqrt{(L+\lambda) \Phi_{n}^{j}} & \hat{\mathbf{x}}_{n-1}^{j}-\sqrt{(L+\lambda) \Phi_{n}^{j}}
\end{array}\right]
$$

where $\Phi$ indicates the covariance, $L$ represents the dimension of the state vector, $\alpha, \beta$ and $\lambda$ are the scaling parameters. By passing the SP through the process equations, it calculates the state and covariance as shown below

$$
\hat{\overline{\mathbf{x}}}_{n \mid n-1}=\sum_{i=0}^{2 L} w_{i}^{s t a} \xi_{n \mid n-1}^{x, i} ; \quad \Phi_{n \mid n-1}=\sum_{i=0}^{2 L} w_{i}^{\mathrm{cov}}\left[\xi_{n \mid n-1}^{x, i}-\hat{\overline{\mathbf{x}}}_{n \mid n-1}\right]\left[\xi_{n \mid n-1}^{x, i}-\hat{\overline{\mathbf{x}}}_{n \mid n-1}\right]^{T}
$$

where the weight parameters are defined for state by $w^{\text {sat }}$ and for covariance via $w^{\text {cov }}$. Additionally, the Kalman gain $\left(\mathbf{K}^{\text {gain }}\right)$ is estimated as

$$
\mathbf{K}_{n}^{\text {gain }}=\Phi_{n}^{x y}\left(\Phi_{n}^{y y}\right)^{-1}
$$

The covariance $(\Phi)$ and cross-covariance $\left(\Phi^{x y}\right)$ are estimated as described below

$$
\Phi_{n}^{y y}=\sum_{i=0}^{2 L} w_{i}^{\mathrm{cov}}\left[\xi_{n \mid n-1}^{x, i}-\hat{\mathbf{y}}_{n \mid n-1}\right]\left[\xi_{n \mid n-1}^{x, i}-\hat{\mathbf{y}}_{n \mid n-1}\right]^{T}, \Phi_{n}^{x y}=\sum_{i=0}^{2 L} w_{i}^{\mathrm{cov}}\left[\xi_{n \mid n-1}^{x, i}-\hat{\mathbf{x}}_{n \mid n-1}\right]\left[\xi_{n \mid n-1}^{x, i}-\hat{\mathbf{y}}_{n \mid n-1}\right]^{T}
$$

Finally, the posterior estimates are calculated as

$$
\hat{\mathbf{\mathbf { x }}}_{n}=\hat{\overline{\mathbf{X}}}_{n \mid n-1}+\mathbf{K}_{n}^{\text {gain }}\left(\mathbf{y}_{n}-\hat{\mathbf{y}}_{n \mid n-1}\right)
$$

In [10], the authors have investigated the nonlinear identification problem by employing the extended Kalman filter (EKF). However, the following work conducted out by [9] has reported the serious drawbacks of the EKF. Further, the applicability and superior performances of UKF has been confirmed by many researches such as [1], [3], [6], [7], [11], [12] and [13].

\section{Numerical Implementations}

The aim here is to identify the damping matrix components as it is often very complicated task to deal with in the area of structural engineering. To this end, an 8-DOFs dynamical system is assumed and the parameters are along with the unknown states are estimated. 


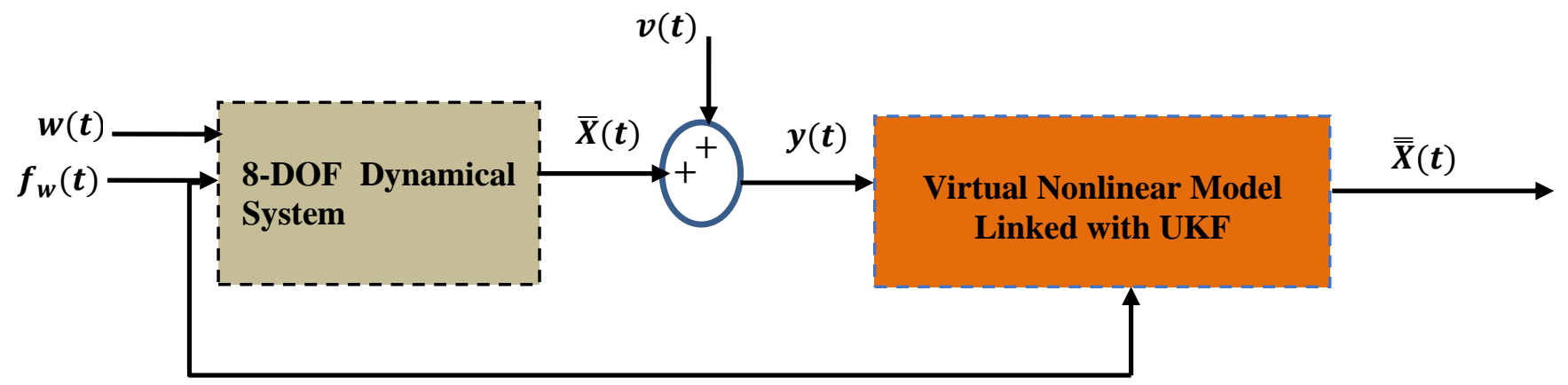

Fig. 2: The schematic view of the problem combined with nonlinear observer.

In order to understand the flow of the studied problem, Figure 2 is provided. The damping matrix was changed/corrupted $\left(C^{\text {new }}\right) 50 \%$ to its original values of $C$, which means $C^{\text {new }}=1.5 \times C$ in order to perform simulations. It can be noticed that the damping matrix has changed significantly to investigates the critical effect of damping. Firstly, the response of the original system is evaluated and presented in Figure 3, where the top floor and the first floors displacement time history is shown in the previous figure.
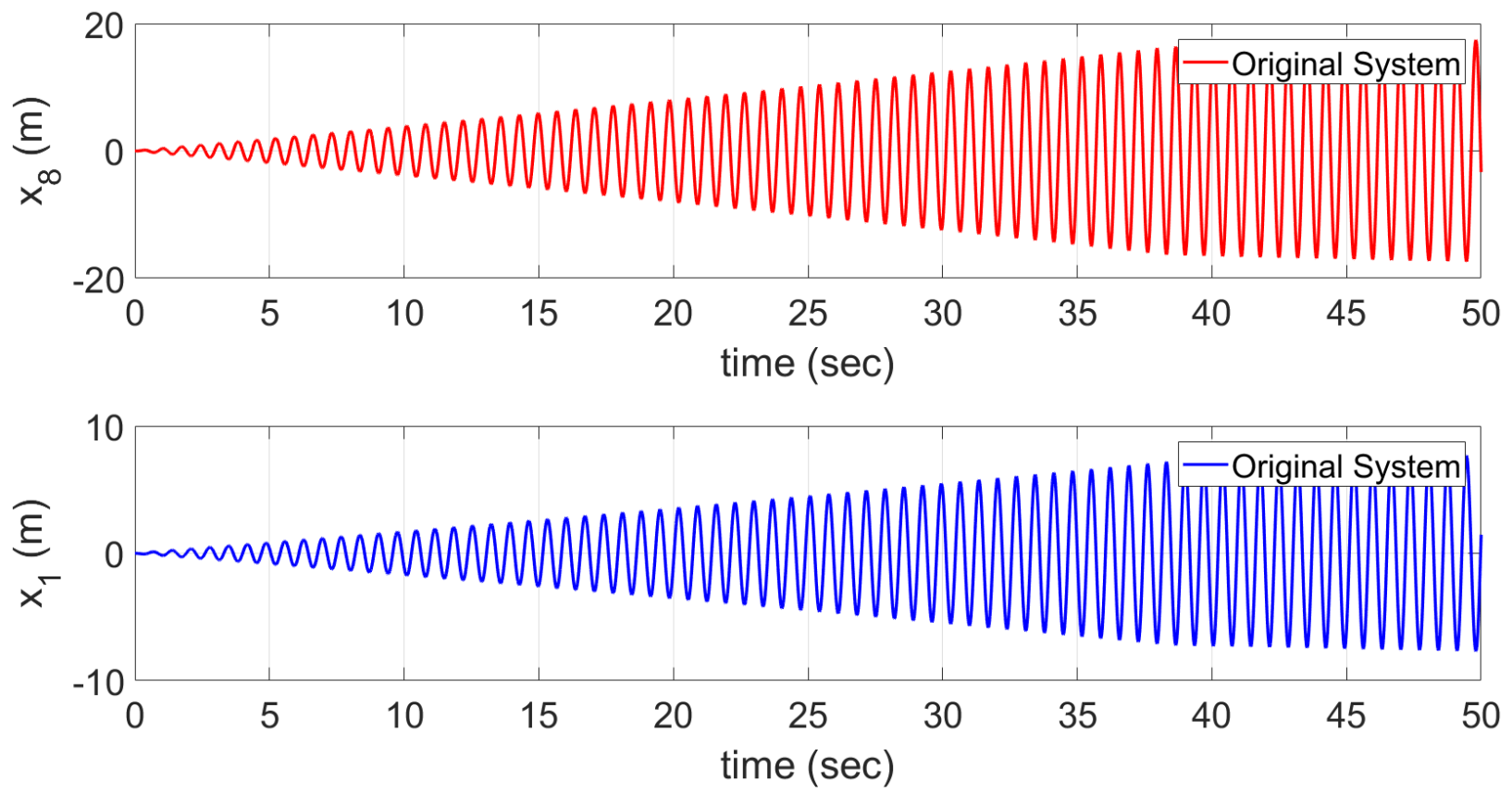

Fig. 3: The top and first floor displacement with everything (system) correct. 

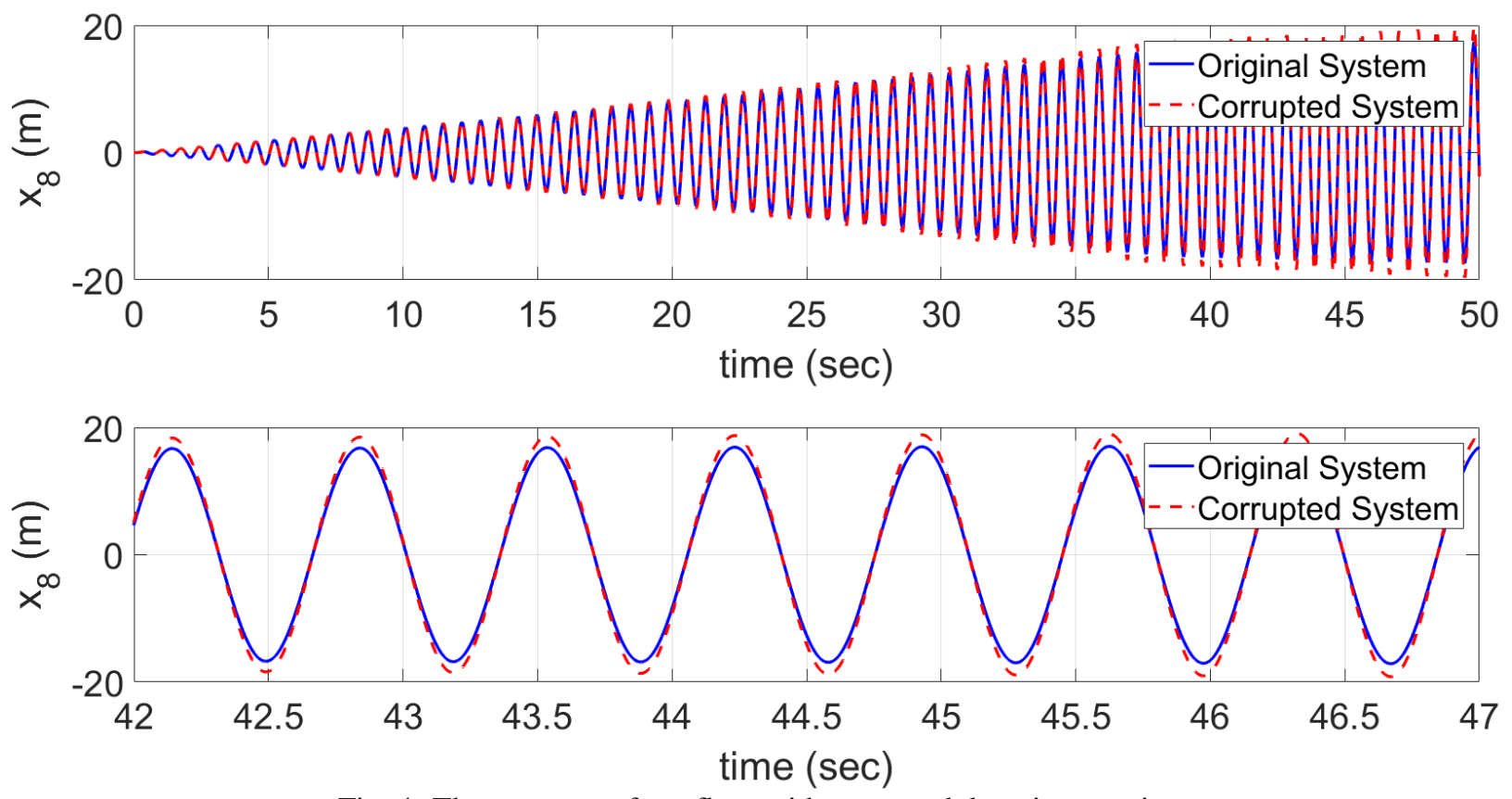

Fig. 4: The response of top floor with corrupted damping matrix.

In order to observe the maximum effect of corrupted damping matrix, the top floor response is evaluated and depicted in Figure 4. It is evident from the figure that there is a change in the amplitude which should be due to the damping. Hence, it is essential to monitor the damping changes of structures as significant change in amplitude may leads to damage. Further, the response of the original system and the system with identified damping matrix is also compared that is shown in Figure 5. From the previous figure, it is clearly visible that the damping was identified with quite high accuracy as the identified system response if matched identically with the original one.
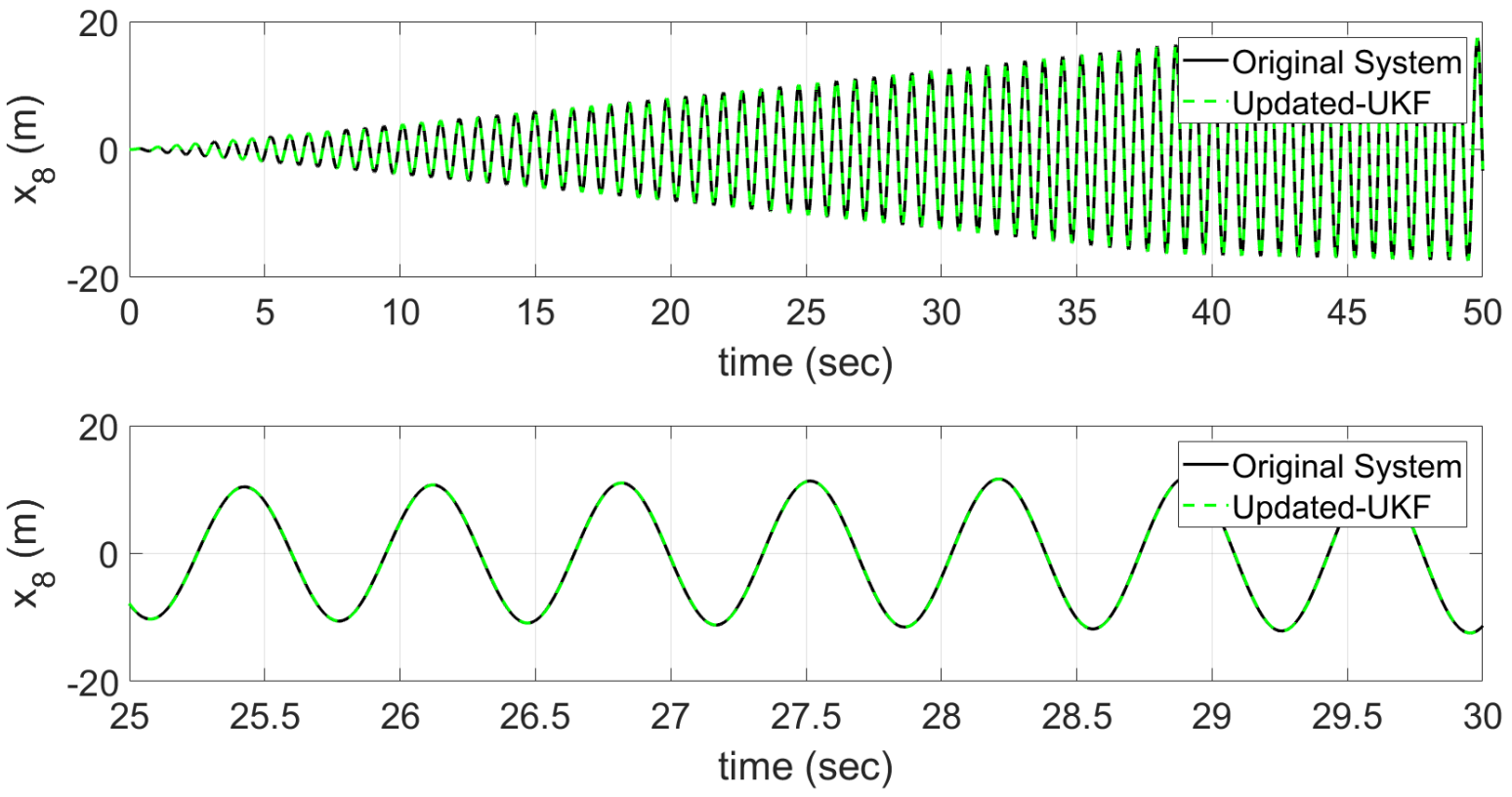

Fig. 5: The top floor displacement with estimated damping matrix via the studied approach. 


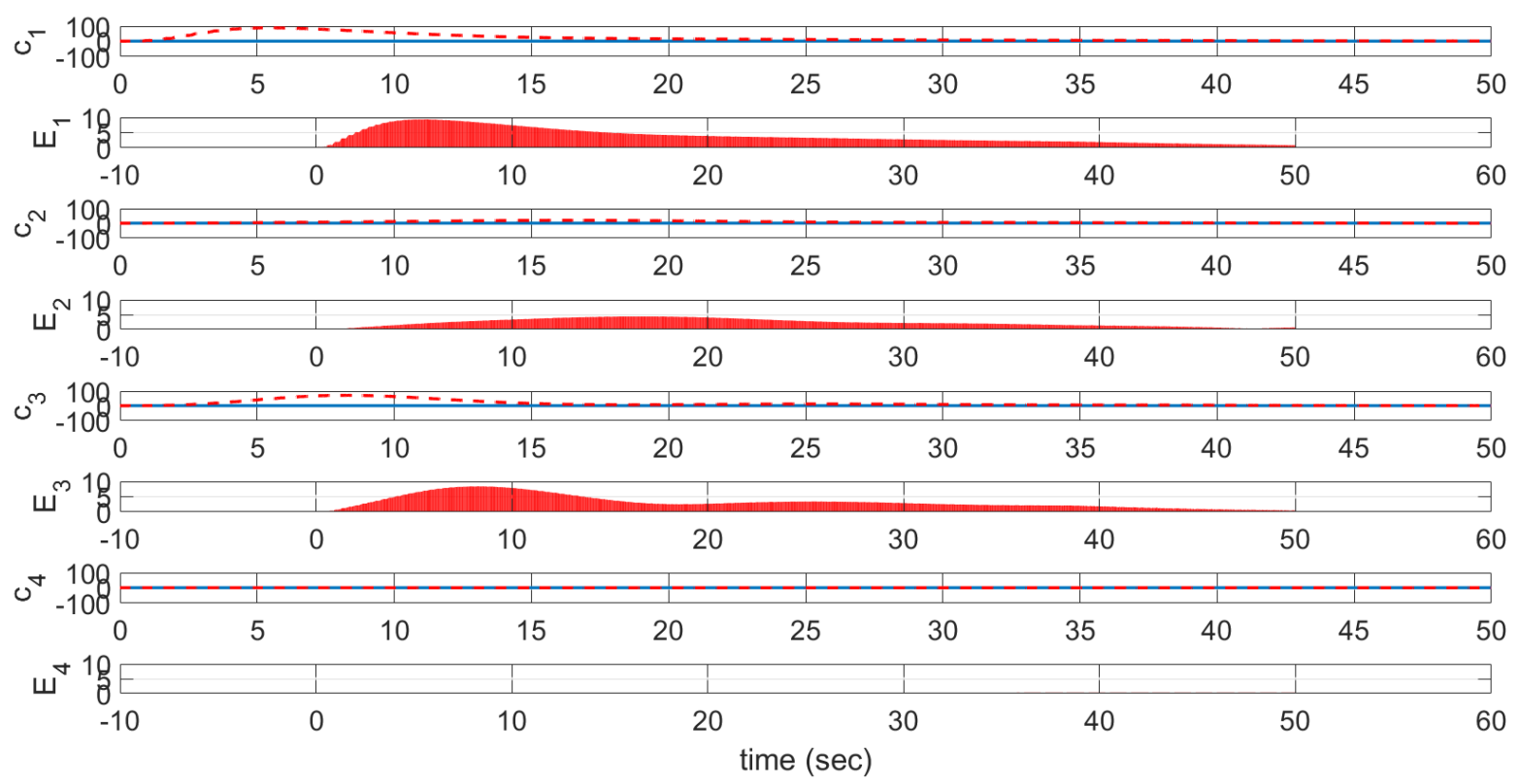

Fig. 6: The estimated damping components $\left(c_{1}-c_{4}\right)$ and the estimated errors $\left(E_{1}-E_{4}\right)$ by the proposed scheme.

The resonant frequencies of the original system and the system with identified damping matrix are compared and an excellent agreement is observed. The frequencies of the original and identified systems are summarized in Table 1. However, it needs to be mentioned that damping does not have serious influence on frequencies. On the other hand, the change in stiffness or mass matrix will lead to serious change in the natural frequencies of the system.

Table 1: Summary of identified damping components.

\begin{tabular}{|c|c|c|}
\hline Number of Floors & $\begin{array}{c}\text { Resonant Frequencies of True } \\
\text { System }(\mathrm{Hz})\end{array}$ & $\begin{array}{c}\text { Resonant Frequencies System } \\
\text { with Identified Damping (Hz) }\end{array}$ \\
\hline 1st & 1.4356 & 1.4356 \\
\hline 2nd & 4.2234 & 4.2233 \\
\hline 3rd & 5.9550 & 5.9550 \\
\hline 4th & 6.7657 & 6.7657 \\
\hline 5th & 8.4217 & 8.4217 \\
\hline 6th & 8.9148 & 8.9148 \\
\hline 7th & 10.5458 & 10.5458 \\
\hline 8th & 11.5640 & 11.5640 \\
\hline
\end{tabular}

Finally, the estimated damping components $\left(c_{1}-c_{8}\right.$ in $\left.\mathrm{N}-\mathrm{s} / \mathrm{m}\right)$ are compared and exhibited in the following Figure 6 and Figure 7. It can be seen that an excellent agreement is observed. Further, the errors $\left(E_{1}-E_{8}\right.$ in $\left.\%\right)$ of the estimated damping components are also calculated and depicted in the early mentioned figures. 


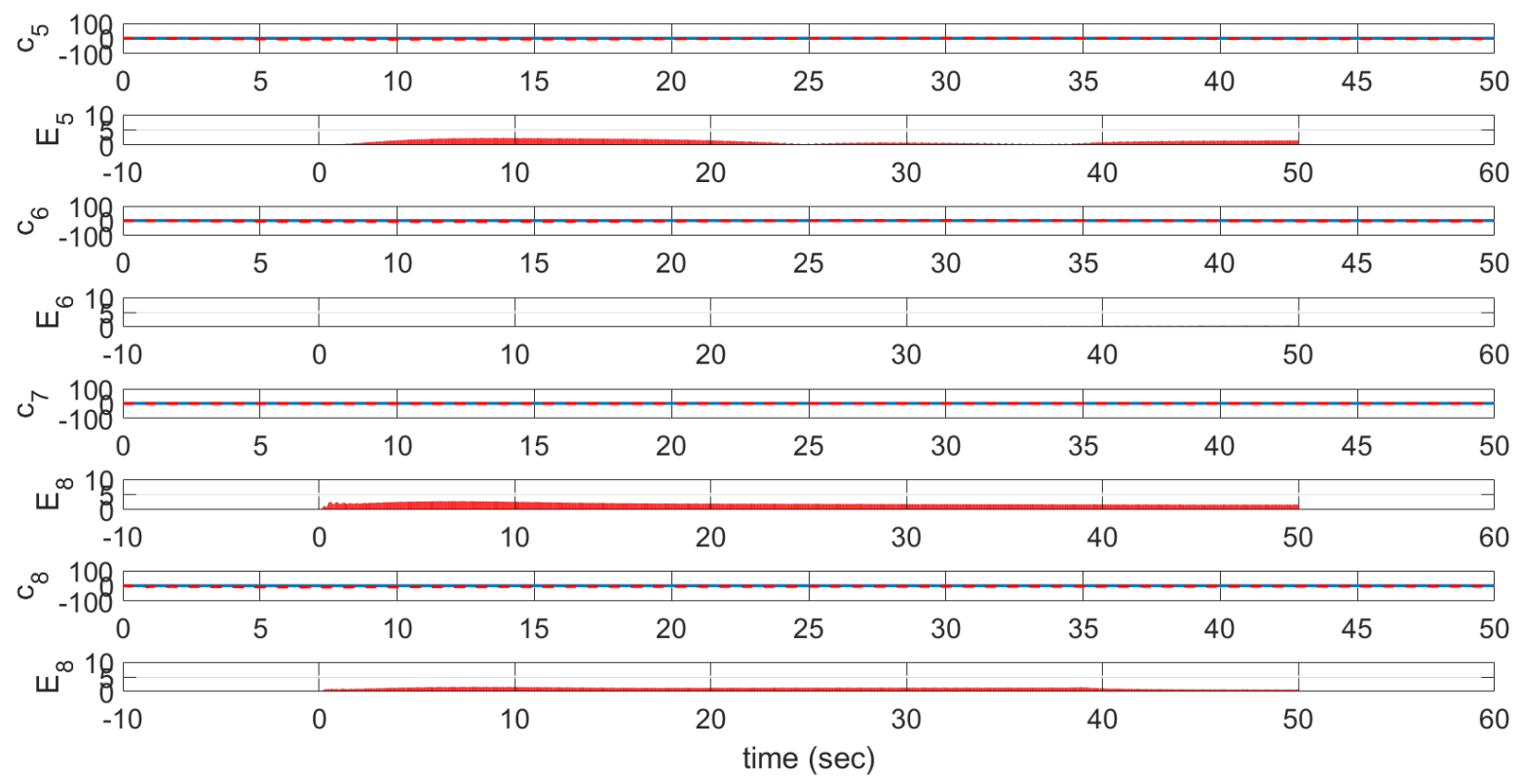

Fig. 7: The estimated damping components $\left(c_{5}-c_{8}\right)$ and the estimated errors $\left(E_{5}-E_{8}\right)$ by the investigated approach.

\section{Conclusion}

The parameter identification problem coupled with structural parameters uncertainty is explored. The uncertainties are inducing from the unknown damping components, process, and measurement noise as well as from initial covariance. This study investigated the identification of damping components of the studied problem and estimates the states numerically by considering an eight DOF system. In short, the following concluding remarks can be made:

- The change in damping matrix may change the amplitude of the displacement, velocity, and acceleration of the system which may lead to damage.

- If the damping matrix is only changed and the mass and stiffness matrices remain constant, then the natural frequencies of the structure may not change significantly.

- The proposed method is capable of identifying the damping matrix quite accurately, as a result, the identified system response quite closely agreed with the true data.

- The identification of damping matrix will assist to optimize the damping of the systems coupled with smart control devices such as magnetorheological (MR) damper.

- The initial covariance of the unknown parameters e.g. damping has critical impact on identification. However, it is recommended that one's should set a reasonable initial covariance. It is observed that the lower values of initial covariance provide a good estimation. However, trial and errors are required to have an optimal starting.

Further study is planned to investigate the control application via active or semi-active control approaches linked with the identification of the damping matrix.

\section{Acknowledgements}

This work is sponsored by the grant number F-003661-553-A1G-1140901 of TU Dresden's Institutional Strategy, Dresden University of Technology-TU Dresden, Dresden, Germany. 


\section{References}

[1] M. S. Miah, "Semi-active control for magnetorheological dampers via coupling of system identification methods," Doctoral Dissertation, ETH-Zürich, Zurich, Switzerland, 22776, 2015.

[2] H. Kosorus, M. Hollrigl-Binder, H. Allmer, and J. Kung, "On the Identification of Frequencies and Damping Ratios for Structural Health Monitoring Using Autoregressive Models," in 23rd International Workshop on Database and Expert Systems Applications, pp. 23-27, 2012.

[3] M. S. Miah, "Structural Parameters Uncertainties Effect on Structural Responses," in Proc. of The Fifth International Conference On Advances in Civil and Structural Engineering - CSE 2016, pp. 37-41, 2016.

[4] N. K. Sinha and M.Y. Tang, "On-line system identification in real time using a minicomputer," Mathematics and Computers in Simulation, vol. 19, no. 2, pp. 113-120, 1975.

[5] C. Ince, and M. Sahin, "Real-time deformation monitoring with GPS and Kalman Filter," Earth Planets and Space, vol. 52, no. 10, pp. 837-840, 2000.

[6] M. Wu, and A. W. Smyth, "Application of the unscented Kalman filter for real-time nonlinear structural system identification," Structural Control and Health Monitoring, vol. 14, pp. 971-990, 2007.

[7] M. S. Miah, E. N. Chatzi, V. K. Dertimanis and F. Weber, "Real-time experimental validation of a novel semi-active control scheme for vibration mitigation," Structural. Control Health Monitoring, vol. 24, pp. e1878, 2017.

[8] J. Uhlmann, "Dynamic Map Building and Localization: New Theoretical Foundations," Ph.D. Thesis, University of Oxford, 1995.

[9] S. J. Julier, and J. Uhlmann, A New Extension of the Kalman Filter to Nonlinear Systems. Int. Symp. Aerospace/Defense Sensing, Simul. and Controls, vol. 3, pp. 1-12, 1997.

[10] L. Jeen-Shang, and Z. Yigong, "Nonlinear structural identification using extended Kalman filter," Computers \& Structures, vol. 52, no. 4, pp. 757-764, 1994.

[11] K. Xiong, H. Y. Zhang, and C. W. Chan, Performance evaluation of UKF-based nonlinear filtering. Automatica, vol. 42, no. 2, pp. 261-270, 2006.

[12] S.-M. Chow, E. Ferrer, and J. R. Nesselroade, An Unscented Kalman Filter Approach to the Estimation of Nonlinear Dynamical Systems Models. Multivariate Behavioral Research, vol. 42, no. 2, pp. 283-321, 2007.

[13] P. Kachroo, K. Ozbay, and A. Narayanan, "Investigating the use of kalman filtering approaches for dynamic origindestination trip table estimation," in Proceedings IEEE Southeastcon '97. 'Engineering the New Century, Blacksburg, USA, pp. 138-142, 1997. 